\title{
Homocysteine-mediated thrombosis and angiostasis in vascular pathobiology
}

\author{
Joseph Loscalzo \\ Department of Medicine, Brigham and Women's Hospital, Harvard Medical School, Boston, Massachusetts, USA.
}

\begin{abstract}
The mechanisms by which homocysteine contributes to atherothrombosis are complex and their in vivo relevance uncertain. In this issue of the JCI, Jacovina and colleagues report a unique in vivo mechanism by which homocysteine may contribute to vascular disease (see the related article beginning on page 3384 ). This group had previously reported that homocysteine impairs endothelial cell surface plasminogen activation by posttranslationally modifying annexin A2, the coreceptor for plasminogen and tissue plasminogen activator. They now show that an annexin A2-deficient mouse rendered hyperhomocysteinemic by dietary means has impaired fibrinolysis, perivascular fibrin persistence, and attenuated angiogenesis (angiostasis). Potential mechanisms by which homocysteine-dependent changes in endothelial phenotype link thrombosis to angiostasis are reviewed and their relationship to homocysteine-dependent vascular disease considered.
\end{abstract}

Over the past 30 years, evidence from many in vitro and in vivo studies supports an association between mild to moderate hyperhomocysteinemia and vascular dysfunction and disease (1). A key mechanism that predisposes to vascular disease in hyperhomocysteinemia is endothelial dysfunction (2). Homocysteine induces endothelial dysfunction in part by promoting oxidant stress, as illustrated in cellular studies $(3,4)$ and in genetic animal models $(2,5,6)$. The mechanisms for homocysteine-induced oxidant stress are complex and include inhibiting the translation of glutathione peroxidase-1 (7), a major antioxidant enzyme in vascular cells that regulates mitochondrial reactive oxygen species flux (8) and whose overexpression rescues the normal vascular phenotype of hyperhomocysteinemic mice (9); upregulation of NADPH oxidase expression (10); enhanced expression of inducible nitric oxide synthase (10) and uncoupling of nitric oxide synthases (11); and decreased glutathione levels owing to decreased cysteine synthesis (12), thereby shifting the redox balance of endothelial cells toward oxidant stress.

Among the specific phenotypic properties of the endothelial cell that go awry in

Conflict of interest: The author serves on scientific advisory boards for BioMarin, N30 Pharma, and HealthCare Ventures.

Citation for this article: J. Clin. Invest. 119:3203-3205 (2009). doi:10.1172/JCI40924. hyperhomocysteinemia are its antithrombotic properties. Hyperhomocysteinemia produces a prothrombotic state (13), some mechanisms for which include enhanced platelet activation, enhanced coagulation (likely as a consequence of increased tissue factor expression; ref. 14), and attenuated fibrinolysis. Two recognized mechanisms for reduced fibrinolysis include posttranslational modification of fibrinogen by homocysteinylation, rendering the fibrin derived from fibrinogen relatively resistant to plasmin (15), and increased activity of thrombin-activatable fibrinolysis inhibitor (TAFI) (16).

In this issue of the JCI, Jacovina and colleagues (17) report the existence of another antifibrinolytic mechanism caused by homocysteine. In the group's earlier work $(18,19)$, they demonstrated that the calcium-regulated, phospholipid-binding protein annexin A2 is an endothelial coreceptor for TPA and plasminogen that facilitates plasminogen activation by enhancing the catalytic efficiency of TPA. They next demonstrated that homocysteine forms a mixed disulfide bond with the Cys9 residue of annexin A2, which decreases the binding affinity of the receptor for TPA. As a result of this posttranslational modification, endothelial cell surface plasminogen activation is markedly attenuated (18). These observations were next confirmed in vivo using Anxa2 $2^{-/-}$mice, which showed increased perivascular fibrin deposition and impaired fibrin clearance. Fibrin formation and plasmin-dependent remodeling are not only important in the hemostatic response to vascular injury, but also for luminal recanalization and angiogenesis during the healing phase. Consistent with this role for fibrin and plasmin, the Anxa2 $2^{-/-}$mice showed significantly impaired angiogenesis in a range of vascular injury models (neoangiogenesis) (19). In their latest study, reported in this issue of the JCI (17), the investigators found that moderate hyperhomocysteinemia induced by feeding mice methionine also leads to impaired fibrinolysis with perivascular fibrin persistence as well as attenuated angiogenesis (angiostasis). In this animal model, they observed significantly reduced TPA-annexin A2 binding and plasminogen activation consistent with the importance of endothelial cell surface plasmin activity for normal fibrin clearance and angiogenesis. The pathophenotype was readily reversed by infusion of wild-type recombinant annexin A2 into the hyperhomocysteinemic mice (17).

\section{Homocysteine and angiostasis}

These interesting data (17) support yet another novel mechanism by which hyperhomocysteinemia promotes the complications of atherothrombotic vascular disease, that is, limiting the angiogenic response to ischemia. Homocysteine-dependent changes in the vascular phenotype previously shown to contribute to impaired angiogenesis include a decrease in glutathione peroxidase expression and consequent increase in oxidant stress, leading to endothelial progenitor cell dysfunction $(7,20)$; decreased bioactive nitric oxide generation $(2,3)$; and dysregulation of MMP activity (21). Specifically, homocysteine has been shown to increase the expression and activity of MMP-2 and MMP-9 (21). In light of the results of the study by Jacovina and colleagues (17), the increase in MMP activity suggests that zymogen activation does not alone depend on cell surface-bound 


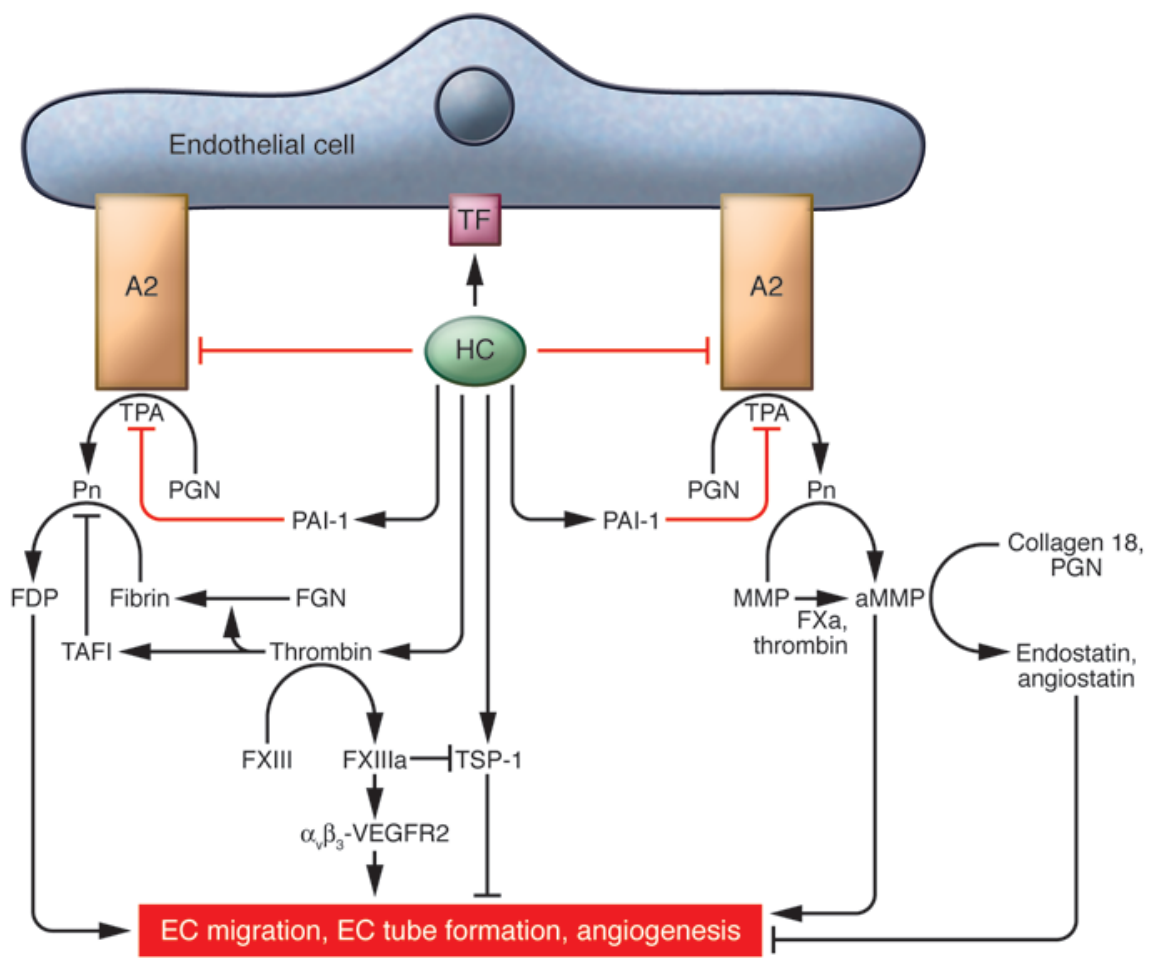

Figure 1

Direct and indirect effects of homocysteine on angiogenesis. Lines with arrows indicate permissive actions, and lines with end bars indicate inhibitory actions. Red lines indicate the contributions of the work of Jacovina and colleagues (17) to these pathways. Homocysteine (HC) induces tissue factor (TF) expression on the endothelial cell and inhibits annexin A2-dependent plasminogen (PGN) activation by TPA. In addition, homocysteine increases plasminogen activator inhibitor-1 (PAl-1) expression to impair further fibrinolysis. By activating tissue factor expression, homocysteine promotes local generation of thrombin, which converts fibrinogen (FGN) to fibrin, impairs fibrinolysis by increasing TAFI, and activates factor XIII (FXIII). FXIIIa, in turn, not only crosslinks fibrin, but also crosslinks the integrin $\alpha_{v} \beta_{3}$ to VEGFR2, which stimulates angiogenesis. Thrombin, along with factor Xa (FXa), activates MMPs (to aMMPs) to promote endothelial cell migration and angiogenesis. Offsetting these indirect angiogenic effects of thrombin are its inhibitory effects, which include factor XIIla-mediated inhibition of thrombospondin-1 (TSP-1) and the generation of angiogenesis inhibitors endostatin and angiostatin by MMPs acting on collagen 18 and plasminogen, respectively. $\alpha_{V} \beta_{3}$-VEGFR2, factor XIIla-crosslinked $\alpha_{v} \beta_{3}$-VEGFR2; A2, annexin A2; FDP, fibrin degradation product; Pn, plasmin.

plasmin; rather, as recently shown in vitro, both Factor $\mathrm{Xa}$ and thrombin can also activate MMP-2 (22), thereby providing a potential mechanism by which to link the prothrombotic effects of homocysteine to matrix degradation. As MMPdependent matrix degradation is essential for (endothelial) cell migration, the association of increased MMP activity with impaired angiogenesis may at first appear paradoxical. However, two angiogenesis inhibitors, endostatin and angiostatin, can be generated by the action of MMPs on collagen 18 and plasminogen, respectively $(23,24)$. Taken together, these data suggest that homocysteine-mediated, tissue factor-dependent thrombin generation not only promotes the prothromstatic role for thrombin. A thrombin-mediated increase in TAFI (16) not only inhibits fibrinolysis, but also inhibits endothelial tube formation and angiogenesis (25). Inhibition of fibrinolysis leads to attenuated release of proteolytic degradation fragments of fibrin that have angiogenic potential (26), such as fibrin fragment $\mathrm{E}$ (27). These indirect angiostatic effects of thrombin may be offset by its activation of the transglutaminase factor XIII; factor
XIIIa not only crosslinks fibrin, but also crosslinks the integrin $\alpha_{V} \beta_{3}$ with VEGFR2 on the endothelial cell to promote angiogenesis and suppresses expression of the natural angiostatic adhesive glycoprotein thrombospondin-1 (28). While these indirect factor XIII-mediated angiogenic effects of thrombin may be active in a hyperhomocysteinemic ischemic model, they are clearly inadequate to overcome the angiostatic effects of homocysteine, leading to impaired angiogenesis in the model.

Lastly, as yet another potential level of regulation of angiogenesis by the homocysteine-dependent prothrombotic state, consider the consequences of elevated homocysteine concentrations on the methylation potential of vascular cells. In general, elevated homocysteine leads to a decrease in the S-adenosyl-methionine/S-adenosyl-homocysteine ratio, which decreases methylation potential and suppresses global DNA methylation (29). As a result, homocysteine can increase the expression of genes that inhibit fibrinolysis, such as plasminogen activator inhibitor-1 (30), and that inhibit angiogenesis, such as thrombospondin-1 (31).

\section{Conclusions}

Thus, the current study by Jacovina and colleagues (17) adds an important new feature to the adverse vascular phenotype of hyperhomocysteinemia, namely, impaired angiogenesis or angiostasis. Furthermore, this study links angiostasis to altered endothelial cell surface plasminogen activation, supporting the importance of plasmin in the angiogenic response. In addition and importantly, their results give one pause to consider the pathobiological network that defines the interface between thrombosis and angiogenesis (see Figure 1). Taken together, these findings highlight the complexity of molecular events that conspire to produce a prothrombotic and angiostatic endothelial pathophenotype from homocysteine exposure and offer novel explanations for the epidemiological association between hyperhomocysteinemia and vascular disease.

\section{Acknowledgments}

This work was supported in part by NIH grants HL61795, HV28178, HL81587, and HL70819. The author wishes to thank Stephanie Tribuna for expert technical assistance.

Address all correspondence to: Joseph Loscalzo, Department of Medicine, Brigham 
and Women's Hospital, 75 Francis Street, Boston, Massachusetts 02115-2394, USA. Phone: (617) 732-6340; Fax: (617) 732-6439; E-mail: jloscalzo@partners.org.

1. Maron, B., and Loscalzo, J. 2009. The treatment of hyperhomocysteinemia. Annu. Rev. Med. 60:39-54.

2. Eberhardt, R.T., et al. 2000. Endothelial dysfunction in a murine model of mild hyperhomocyst(e)inemia. J. Clin. Invest. 106:483-491.

3. Upchurch, G.R., Jr., et al. 1997. Homocyst(e)ine decreases bioavailable nitric oxide by a mechanism involving glutathione peroxidase. J. Biol. Chem. 272:17012-17017.

4. Heydrick, S.J., et al. 2004. L-Homocysteine and L-homocystine stereospecifically induce endothelial nitric oxide synthase-dependent lipid peroxidation in endothelial cells. Free Radic. Biol. Med. 36:632-640.

5. Dayal, S., and Lentz, S.R. 2008. Murine models of hyperhomocysteinemia and their vascular phenotypes. Arterioscler. Thromb. Vasc. Biol. 28:1596-1605.

6. Dayal, S., and Lentz, S.R. 2007. Role of redox reactions in the vascular phenotype of hyperhomocysteinemic animals. Antioxid. Redox Signal. 9:1899-1909.

7. Handy, D.E., Zhang, Y., and Loscalzo, J. 2005. Homocysteine downregulates glutathione peroxidase (GPx1) by decreasing translation. J. Biol. Chem. 280:15518-15525.

8. Handy, D.E., et al. 2009. Glutathione peroxidase-1 regulates mitochondrial function to modulate redox-dependent cellular responses. J. Biol. Chem. 284:11913-11921.

9. Weiss, N., Zhang, Y.Y., Heydrick, S., Bierl, C., and Loscalzo, J. 2001. Overexpression of cellular glutathione peroxidase rescues homocyst(e)ineinduced endothelial dysfunction. Proc. Natl. Acad. Sci. U. S. A. 98:12503-12508

10. Ungvari, Z., et al. 2003. Increased superoxide production in coronary arteries of hyperhomocysteinemia: role of tumor necrosis factor-alpha, $\mathrm{NAD}(\mathrm{P}) \mathrm{H}$ oxidase, and inducible nitric oxide synthase.
Arterioscler. Thromb. Vasc. Biol. 23:418-424.

11. Topal, G., et al. 2004. Homocysteine induces oxidative stress by uncoupling of NO synthase activity through reduction of tetrahydrobiopterin. Free Radic. Biol. Med. 36:1532-1541.

12. Vitvitsky, V., et al. 2004. Perturbations in homocysteine-linked redox homeostasis in a murine model for hyperhomocysteinemia. Am. J. Physiol. Regul. Integr. Comp. Physiol. 287:R39-R46.

13. Dayal, S., et al. 2005. Enhanced susceptibility to arterial thrombosis in a murine model of hyperhomocysteinemia. Blood. 108:2237-2243.

14. Undas, A., Brozek, J., and Szczeklik, A. 2005. Homocysteine and thrombosis: from basic science to clinical evidence. Thromb. Haemost. 94:907-915.

15. Sauls, D.L., et al. 2006. Modification of fibrinogen by homocysteine thiolactone increases resistance to fibrinolysis: a potential mechanism of the thrombotic tendency in hyperhomocysteinemia. Biochemistry. 45:2480-2487.

16. Colucci, M., et al. 2008. Mild hyperhomocysteinemia is associated with increased TAFI levels and reduced plasma fibrinolytic potential. J. Thromb. Haemost. 6:1571-1577.

17. Jacovina, A.T., et al. 2009. Homocysteine inhibits neoangiogenesis in mice through blockade of annexin A2-dependent fibrinolysis. J. Clin. Invest. 119:3384-3394.

18. Hajjar, K.A., et al. 1998. Tissue plasminogen activator binding to the annexin II tail domain. Direct modulation by homocysteine. J. Biol. Chem. 273:9987-9993.

19. Ling, Q., et al. 2004. Annexin II regulates fibrin homeostasis and neoangiogenesis in vivo. J. Clin. Invest. 113:38-48.

20. Galasso, G., et al. 2006. Impaired angiogenesis in glutathione peroxidase-1-deficient mice is associated with endothelial progenitor cell dysfunction. Circ. Res. 98:254-261.

21. Kundu, S., et al. 2009. Nitrotyrosinylation, remodeling and endothelial-myocyte uncoupling in iNOS, cystathionine beta synthase (CBS) knock- outs and iNOS/CBS double knockout mice. J. Cell. Biochem. 106:119-126.

22. Koo, B.-H., Park, M.Y., Jeon, O.-H., and Kim, D.-S. 2009. Regulatory mechanism of MMP-2 enzymatic activity by factor Xa and thrombin. J. Biol. Chem. 284:23375-23385.

23. O'Reilly, M.S., et al. 1997. Endostatin: an endogenous inhibitor of angiogenesis and tumor growth. Cell. 88:277-285.

24. Sodha, N.R., et al. 2009. Endostatin and angiostatin are increased in diabetic patients with coronary artery disease and associated with impaired coronary collateral formation. Am. J. Physiol. Heart Circ. Physiol. 296:H428-H434.

25. Guimarães, A.H., et al. 2007. TAFI and pancreatic carboxypeptidase B modulate in vitro capillary tube formation by human microvascular endothelial cells. Arterioscler. Thromb. Vasc. Biol. 27:2157-2162.

26. Romer, J., et al. 1996. Impaired wound healing in mice with a disrupted plasminogen gene. Nat. Med. 2:287-292.

27. Bootle-Wilbraham, C.A., Tazzyman, S., Thompson, W.D., Stirk, C.M., and Lewis, C.E. 2001. Fibrin fragment E stimulates proliferation, migration and differentiation of human microvascular endothelial cells in vitro. Angiogenesis. 4:269-275.

28. Dardik, R., Loscalzo, J., Eskaraev, R., and Inbal, A. 2005. Molecular mechanisms underlying the proangiogenic effect of factor XIII. Arterioscler. Thromb. Vasc. Biol. 25:526-532.

29. Ingrosso, D., and Perna, A.F. 2009. Epigenetics in hyperhomocysteinemic states: a special focus on uremia. Biochim. Biophys. Acta. 1790:892-899.

30. Gao, S., Skeldal, S., Krogdahl, A., Sorensen, J.A., and Andreasen, P.A. 2005. CPG methylation of the PAI-1 gene 5'-flanking region is inversely correlated with PAI-1 mRNA levels in human cell lines. Thromb. Haemost. 94:651-660.

31. Hu, C.J., et al. 2006. Promoter region methylation and reduced expression of thrombospondin-1 after oxygen deprivation in murine cerebral endothelial cells. J. Cereb. Blood Flow Metab. 26:1519-1526.

\section{The APCs of neuroprotection}

\section{Charles T. Esmon ${ }^{1}$ and Jonathan D. Glass ${ }^{2}$}

${ }^{1}$ Cardiovascular Biology Research Program, Oklahoma Medical Research Foundation, and Howard Hughes Medical Institute, Oklahoma City, Oklahoma, USA. ${ }^{2}$ Center for Neurodegenerative Disease, Emory University School of Medicine, Atlanta, Georgia, USA.

\begin{abstract}
Mutations in the enzyme superoxide dismutase 1 (SOD1) have been linked to the neurodegenerative disease amyotrophic lateral sclerosis (ALS). In this issue of the JCI, Zhong et al. report that the endogenous anticoagulant activated protein C (APC) is able to cross the blood-spinal cord barrier in mice and signal to both neuronal and non-neuronal cells (see the related article beginning on page 3437 ). This signaling resulted in the suppression of mutant SOD1 synthesis and retarded disease progression in a murine model of ALS. Here we discuss the potential importance of these data and possible relevance to human neurodegenerative diseases.
\end{abstract}

Amyotrophic lateral sclerosis (ALS) is a devastating neurodegenerative disease that strikes in midlife, causing progres-

Conflict of interest: The authors have declared that no conflict of interest exists.

Citation for this article: J. Clin. Invest. 119:3205-3208 (2009). doi:10.1172/JCI40682. sive weakness, disability, and death. Unfortunately, the cause of ALS is unknown, and the treatment is largely palliative. Research into the pathogenesis of and potential treatments for ALS focuses heavily on experimental models that use human genetic mutations in transgenic animals or on cellular mod- els that express mutant proteins known to impart a high risk of developing ALS in people who carry these mutations. Mutant superoxide dismutase 1 (SOD1) is the most common protein known to cause ALS in humans; however, the mechanisms underlying mutant SOD1-related ALS are unknown. People with mutant SOD1-related ALS represent only about $20 \%$ of inherited (i.e., familial) ALS cases and about $2 \%$ of all patients with ALS (1). Nevertheless, the animal models of mutant SOD1-related ALS develop a neurological disorder that mimics the human disease, and investigations using these models have taught us a lot about motor neuron biology as well as the potential interactions between motor 